\title{
Direct Observation of Multiple Pathways of Single-Stranded DNA Stretching
}

\author{
Wuen-shiu Chen, ${ }^{1}$ Wei-Hung Chen, ${ }^{1,2}$ Zephan Chen, ${ }^{1}$ Ashton A. Gooding, ${ }^{1}$ Kuan-Jiuh Lin, ${ }^{2}$ and Ching-Hwa Kiang ${ }^{1, *}$ \\ ${ }^{1}$ Department of Physics and Astronomy, Rice University, Houston, Texas 77005, USA \\ ${ }^{2}$ Department of Chemistry, National Chung Hsing University, Taichung, Taiwan, Republic of China
}

(Received 11 June 2010; published 19 November 2010)

\begin{abstract}
We observed multiple pathways of stretching single-stranded polydeoxynucleotides, poly(dA). Poly(dA) has been shown to undergo unique transitions under mechanical force, and such transitions were attributed to the stacking characteristics of poly(dA). Using single-molecule manipulation studies, we found that poly $(\mathrm{dA})$ has two stretching pathways at high forces. The previously observed pathway has a free energy that is less than what is expected of single-stranded DNA with a random sequence, indicating the existence of a novel conformation of poly(dA) at large extensions. We also observed stepwise transitions between the two pathways by pulling the molecule with constant force, and found that the transitions are cooperative. These results suggest that the unique mechanical property of poly $(\mathrm{dA})$ may play an important role in biological processes such as gene expression.
\end{abstract}

Single-stranded DNA (ssDNA) plays an important role during many of the biological processes such as transcription [1], replication [2], and DNA repair [3], and deregulation of these processes is critical to the development of human cancer [4]. The flexibility of ssDNA facilitates DNA-ligand recognition, a key step in transcription regulation. Furthermore, the stretched form of ssDNA may be important in determining the energetics of DNA recombination [5]. Different secondary structures of ssDNA also indicate different binding affinities [6,7] and thus drew substantial attention [8-11]. However, unlike doublestranded DNA (dsDNA), whose secondary structures of force dependence and dynamics have been extensively studied [12-24], the structure and dynamics of ssDNA is less understood. In some cases, when ssDNA is confined in a nanopore [25] and under force, it adopts a different secondary structure [26]. Moreover, the conformation and dynamics of ssDNA may be similar to that of RNA, which is important in areas such as viral genome packaging [27]. Recently, the force curves of poly(dA) and poly(A) have shown distinct plateaus in overstretching transitions [8], indicating diverse secondary structures at large extensions. However, the nature of the unique transition is still unknown. It was originally speculated that it may be from transition between sugar pucker conformations, though the hypothesis cannot be explained by the known ground state conformation of poly(dA). Here we describe our finding that poly $(\mathrm{dA})$ stretching has two pathways under high forces. One pathway is similar to that of ssDNA with a random sequence, whereas the other pathway has an additional transition that is energetically favored. We also observed stepwise transitions between these two pathways when we stretched them under constant force, and have characterized the kinetic behavior and quantified the energy barrier between these pathways. The results suggest that $\operatorname{poly}(\mathrm{dA})$ has a novel conformation when highly stretched, and such conformation makes poly $(\mathrm{dA})$ more stable at large extensions.

We performed single-molecule measurements of stretching and relaxation of poly $(\mathrm{dA})$ in fluids at room temperature using an atomic force microscope (AFM). $60 \mu \mathrm{l}$ solution containing $10 \mu \mathrm{g} / \mathrm{ml}$ single-stranded poly(dA) (Sigma) prepared in Tris-EDTA buffer $(10 \mathrm{mM}$ Tris-HCl, $1 \mathrm{~m} M$ EDTA, $p \mathrm{H} \mathrm{8)}$ with $150 \mathrm{~m} M \mathrm{NaCl}$ was allowed to absorb onto a freshly exposed gold substrate surface. The sample was incubated for 3 to 5 hours and then rinsed with Tris-EDTA buffer solution to remove unbound poly $(\mathrm{dA})$. The spring constant of the cantilever (MLCT Veeco Probes), determined using the equipartition theorem $[28,29]$, was $0.03-0.05 \mathrm{~N} / \mathrm{m}$. We performed repeated stretching and relaxation cycles and recorded the force as a function of time at a sampling rate of $10 \mathrm{kHz}$. A waiting time of 60 seconds between stretching and relaxation was used to allow poly $(\mathrm{dA})$ to equilibrate before relaxation unless otherwise specified.

We first stretched poly(dA) at constant velocities, ranging from $40 \mathrm{~nm} / \mathrm{s}$ to $2000 \mathrm{~nm} / \mathrm{s}$, followed by a waiting period of 10 to 70 seconds when the position of the cantilever level was held constant, and the molecule was subsequently relaxed to its original state at the same velocity as the stretching cycle [Fig. 1(a)]. We observed two pathways at forces higher than $110 \mathrm{pN}$. In the low-energy pathway $\mathrm{L}$, the force-extension curve has plateaus at $23 \mathrm{pN}$ and $114 \mathrm{pN}$, consistent with the curves observed in Ref. [8]. The force-extension curves were normalized such that at $600 \mathrm{pN}$, the distance between adjacent bases is $0.7 \mathrm{~nm}$ [8]. In addition to pathway L, we observed another pathway, which requires higher free energy, and is denoted as pathway $\mathrm{H}$. Pathway $\mathrm{H}$ has the same plateau at $23 \mathrm{pN}$ but lacks a well-defined second plateau at $110 \mathrm{pN}$. At high pulling velocity, stretching typically follows pathway $\mathrm{H}$, with occasional sudden transitions from 

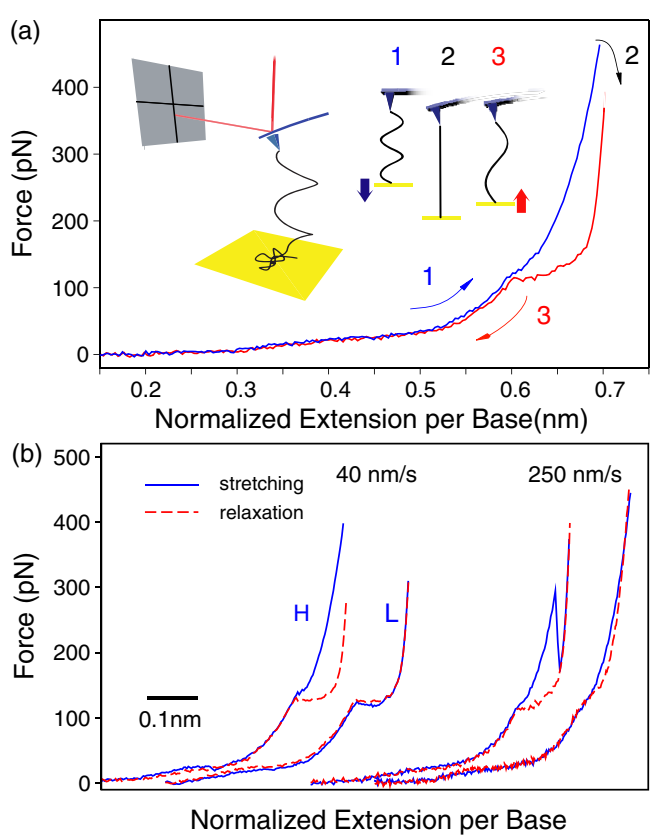

FIG. 1 (color online). Multiple stretching pathways of poly(dA). (a) Force-extension curve of a stretch-relaxation cycle. Inset: One end of poly(dA) was attached to the cantilever tip and the other end to a gold substrate. (b) The first plateau is independent of pulling velocity, whereas the second plateau has two distinct pathways: a high-energy pathway $\mathrm{H}$ and a low-energy pathway L. Shown are two representative cycles at two different pulling velocities.

pathways $\mathrm{H}$ to $\mathrm{L}$ [Fig. 1(b)]. On the other hand, the relaxation curves often show hysteresis, which depends on the length of the waiting time between stretching and relaxation. A waiting time of 60 seconds usually results in the relaxation curve following pathway $\mathrm{L}$, whereas a shorter waiting time typically results in curves that deviate toward pathway $\mathrm{H}$. At low pulling velocities, stretching and relaxation curves both follow pathway $\mathrm{L}$.

To estimate the free energy difference between pathways $\mathrm{H}$ and $\mathrm{L}$, we calculated the area enclosed by the two pathways (area 1 in Fig. 2), and obtained a free energy difference of $1.2 \mathrm{kcal} / \mathrm{mol}$ at a separation of $0.7 \mathrm{~nm}$ per base. Pathway $\mathrm{H}$ is similar to other ssDNA forceextension curves commonly observed, such as $\lambda$-phage ssDNA [5] and poly(dT) [8]. This indicated that molecules following pathway $\mathrm{H}$ are random coils, whereas molecules following pathway $\mathrm{L}$ have additional interactions between bases, perhaps by forming a loosely stacked structure. The structure is likely to have higher bending and stretch modulus compared to the random coil conformation [30]. The area under the second plateau of pathway L (area 2) is $0.9 \mathrm{kcal} / \mathrm{mol}$, which is consistent with theoretical calculations [30].

The observation of multiple pathways of poly(dA) stretching is surprising. The unique pathway $\mathrm{L}$, which is not present in ssDNA of other sequences, is more stable than a random coil ssDNA (pathway $\mathrm{H}$ ). This phenomenon

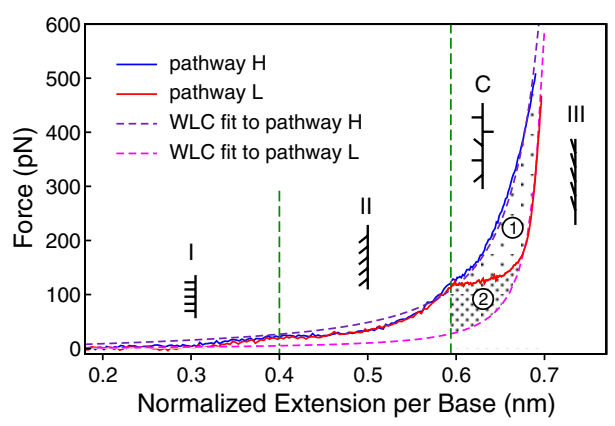

FIG. 2 (color online). The force-extension curve of poly(dA) can be separated into three sections (I, II, and III), and section III has two pathways. Shaded areas 1 and 2 indicate free energy difference (see text).

suggested that poly (dA) has a unique structure when highly stretched, specifically at a per base distance of $0.6 \mathrm{~nm}$ or higher, and the structure stabilizes at $0.7 \mathrm{~nm}$, a distance where the ssDNA is nearly fully stretched. The results suggest that poly(dA) started from a stacked state I, and transitioned to a different stacked state II beginning at $0.34 \mathrm{~nm}$. When stretched beyond $0.6 \mathrm{~nm}$, ploy(dA) may follow (i) pathway $\mathrm{H}$ and become a random coil (ensemble C), or (ii) pathway L and become state III, probably weakly stacked. The order parameter is stacking, whereby the neighboring base interaction lowers the free energy of that conformation. For comparison, we fitted the wormlike-chain (WLC) model [12] to poly(dT) and pathways $\mathrm{H}$ and $\mathrm{L}$ of poly $(\mathrm{dA})$. The fitted WLC parameters for pathway $\mathrm{H}$ and poly(dT) are nearly identical. On the other hand, the persistence lengths for poly(dT) and poly $(\mathrm{dA})$ are $0.2 \mathrm{~nm}$ and $1.2 \mathrm{~nm}$ (uncertainty $0.1 \mathrm{~nm}$ ), respectively, whereas their contour lengths are similar $(0.79 \mathrm{~nm}$ and $0.73 \mathrm{~nm})$. The result suggests that the A base interaction also increases the stiffness of poly $(\mathrm{dA})$, resulting in a larger persistence length.

The highly stretched conformation of poly(dA) state II is stable at $0.6 \mathrm{~nm}$, an extension about $75 \%$ larger than $B$-DNA (Fig. 3). This is similar to the $S$-DNA, a highly

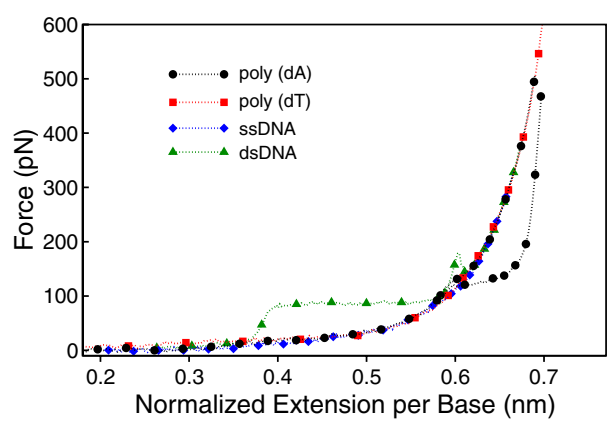

FIG. 3 (color online). Pulling curves for poly(dA), poly(dT), and $\lambda$-phage ssDNA and dsDNA. Pathway $\mathrm{H}$ of poly(dA) coincides with poly(dT) and ssDNA, which are random coils. The transition of poly(dA) also resembles that of dsDNA overstretching transitions. 
stretched form of dsDNA, or $P$-DNA, where the bases are exposed [31]. In addition, the transition point where the two pathways of poly $(\mathrm{dA})$ start to deviate $(0.6 \mathrm{~nm})$ coincides with the onset of $S$-DNA to ssDNA transition [5,32-34]. This suggests that a similar configuration that heightened the $B-S$ transition in dsDNA may also be responsible for the unusual poly $(\mathrm{dA})$ transition.

The existence of pathway $\mathrm{H}$ is facilitated by kinetic trap near $0.6 \mathrm{~nm}$. The force-extension curves of pathway $\mathrm{H}$ overlap well with poly(dT) and $\lambda$-phage ssDNA, with the exception of the kink at $0.6 \mathrm{~nm}$, where poly $(\mathrm{dA})$ transitions from stacked state II to random coil C. However, $\lambda$-phage and poly $(\mathrm{dT})$ are unstacked; they start as random coils and stretching does not result in phase transition. We noticed that a higher pulling velocity usually results in the trajectory following pathway $\mathrm{H}$. More than $90 \%$ of the 46 curves pulled at $2000 \mathrm{~nm} / \mathrm{s}$ follow pathway $\mathrm{H}$, compared to only $10 \%$ of the 45 curves at $40 \mathrm{~nm} / \mathrm{s}$. The existence of the kinetic barrier indicates that pathway $\mathrm{H}$ is metastable.

To further quantify the kinetic barriers of transition from pathways $\mathrm{H}$ to $\mathrm{L}$, we performed constant force experiments $[35,36]$ of poly(dA) under high forces. Single poly(dA) was first stretched at constant velocity until the force reached the desired force, ranging between 50 and $600 \mathrm{pN}$, and the feedback loop was turned on to maintain constant force while the end-to-end distance was recorded as a function of time [Fig. 4(a)]. Figure 4(b) shows representative trajectories of constant force time traces. We observed stepwise transitions from pathways $\mathrm{H}$ to $\mathrm{L}$. The length of poly $(\mathrm{dA})$ increases with time, which indicates a segment of poly(dA) hopped from pathways $\mathrm{H}$ to $\mathrm{L}$. This implies that the transition from random coil to the unique poly $(\mathrm{dA})$ pathway $\mathrm{L}$ is cooperative. The segment length, calculated from the step size, ranges from a few hundred to a few thousand bases. Reverse transitions from pathway $\mathrm{L}$ back to pathway $\mathrm{H}$ have occasionally been observed [inset in Fig. 4(b)].

To obtain the reaction rate constant for the transition from pathways $\mathrm{H}$ to $\mathrm{L}$, we averaged the time traces of a given force [37], as shown in Fig. 4(c). Since this is a unimolecular reaction, we assumed a first order transition and fit the averaged time trace to

$$
x=1-e^{-k(F) t}
$$

where $x$ is the fraction of bases in pathway $\mathrm{L}, k(F)$ is the rate constant at fixed force $F$, and $t$ is time. To estimate the free-energy barrier to hopping, we used the Arrhenius equation

$$
k(F)=A e^{-\Delta G(F) / k_{B} T}
$$

where $\Delta G(F)$ is the energy barrier from pathways $\mathrm{H}$ to $\mathrm{L}$, $k_{B}$ is the Boltzmann constant, $T$ is temperature, and $A$ is the preexponential factor.

Figure 4(c) shows the trace averages of the extension curves under different forces. The transition rate from pathways $\mathrm{H}$ to $\mathrm{L}$ increases with force. The metastable pathway $\mathrm{H}$ is stable over several seconds even at a force

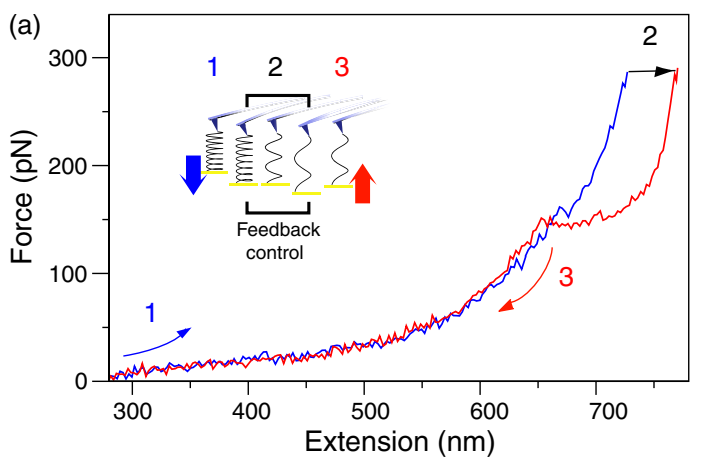

(b)
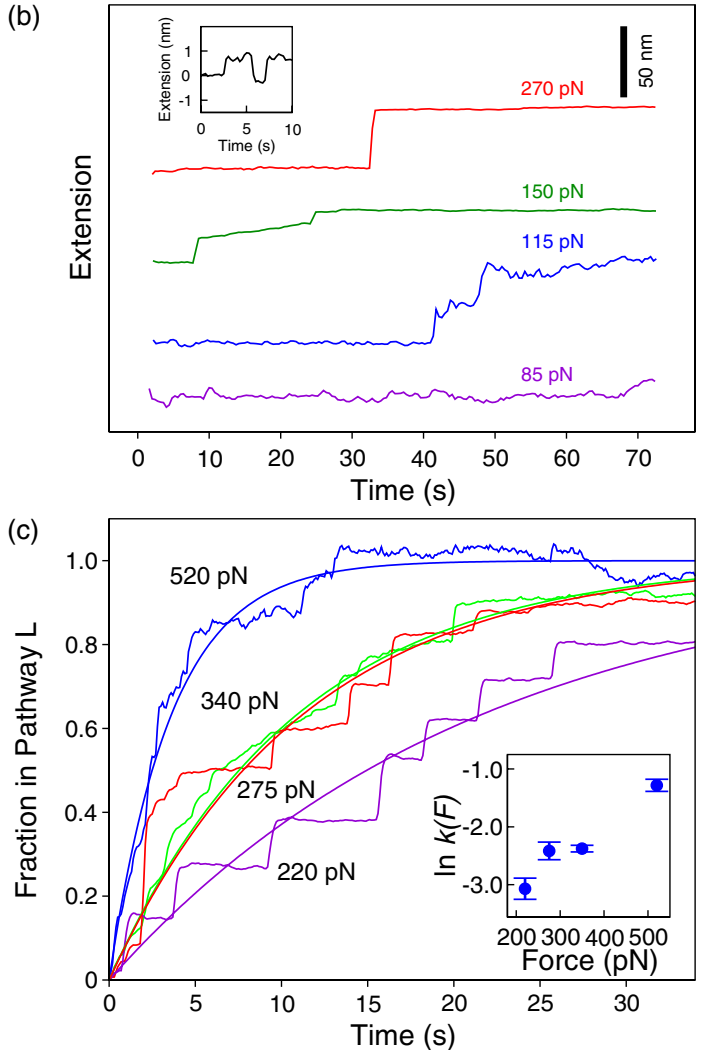

FIG. 4 (color online). Constant force measurement of transition between pathways $\mathrm{H}$ and $\mathrm{L}$ of poly(dA). (a) We pulled poly(dA) at constant velocity until it reached the desired force (step 1), and then turned on the feedback to keep the molecule under constant force while monitoring the end-to-end distance (step 2). Finally, we relaxed the molecule to its original extension (step 3). (b) Typical time traces of poly(dA) at constant force. Inset: reverse transition from pathways $\mathrm{L}$ to $\mathrm{H}$, which occurs more often at high force where the transition barrier is lower. (c) Averaged transition curves between pathways $\mathrm{H}$ and L. The lines are fit to Eq. (1). Inset: Force dependence of the transition rate.

higher than $600 \mathrm{pN}$. The average extension curves are normalized so that the $y$ axis represents the fraction of bases in pathway L. Inset in Fig. 4(c) shows the force dependence on transition rate, where the error was calculated by the bootstrap method. Assuming $A \approx 10^{5} \mathrm{~s}^{-1}$ $[38,39]$, we determined that the force dependent energy barrier ranges from $8.6 \mathrm{kcal} / \mathrm{mol}$ to $7.5 \mathrm{kcal} / \mathrm{mol}$. 
The unique conformational transition of $\operatorname{poly}(\mathrm{dA})$ is consistent with many of the exceptional mechanical properties, resulting from its distinctive segments of repeated A bases called poly $(\mathrm{dA})$ tract. For example, poly $(\mathrm{dA})$ tract regions of DNA are bent and have a reduced helical repeat, which is important in a variety of biological processes such as gene regulation [40]. In addition, the polyA tail, a stretch of A bases in RNA, plays an important role in mRNA stability [41]. Therefore, the unique property of single-stranded A-rich nucleic acids is thought to be a potential drug target for cancer therapy by blocking these sites for gene expression [42,43]. Single-molecule force measurement provides a way to quantify the elastic properties of these segments, and may ultimately be developed into a tool for drug screening.

In summary, we directly observed two stretching pathways in poly $(\mathrm{dA})$. The elasticity of the high-energy pathway $\mathrm{H}$ suggested that the metastable pathway at large extension is a random coil. The low-energy pathway L of poly(dA) may be a novel DNA conformation [44] that contains strong interaction between the adjacent A bases. We have also observed cooperative transitions from highto low-energy pathways using constant force experiments, and estimated the free energy difference between the two pathways. The low-energy pathway L suggests a poly(dA) conformation that may be an important intermediate during many of the biological processes.

We thank NSF DMR-0907676, Welch Foundation No. C-1632, and Hamill Innovation Fund for support.

*chkiang@rice.edu

[1] G. J. L. Wuite, S. B. Smith, M. Young, D. Keller, and C. Bustamante, Nature (London) 404, 103 (2000).

[2] J.-B. Lee, R. K. Hite, S. M. Hamdan, X. S. Xie, C.C. Richardson, and A. M. van Oijen, Nature (London) 439, 621 (2006).

[3] L. Zou and S. J. Elledge, Science 300, 1542 (2003).

[4] D. T. Braddock, J. M. Louis, J. L. Baber, D. Levens, and G. M. Clore, Nature (London) 415, 1051 (2002).

[5] S. B. Smith, Y. J. Cui, and C. Bustamante, Science 271, 795 (1996).

[6] E. Biet, J.-S. Sun, and M. Dutreix, Nucleic Acids Res. 27, 596 (1999).

[7] R. Bar-Ziv and A. Libchaber, Proc. Natl. Acad. Sci. U.S.A. 98, 9068 (2001).

[8] C. Ke, M. Humeniuk, H. S.-Gracz, and P.E. Marszalek, Phys. Rev. Lett. 99, 018302 (2007).

[9] N. L. Goddard, G. Bonnet, O. Krichevsky, and A. Libchaber, Phys. Rev. Lett. 85, 2400 (2000).

[10] M.-N. Dessinges, B. Maier, Y. Zhang, M. Peliti, D. Bensimon, and V. Croquette, Phys. Rev. Lett. 89, 248102 (2002).

[11] M. C. Murphy, I. Rasnik, W. Cheng, T. M. Lohman, and T. Ha, Biophys. J. 86, 2530 (2004).

[12] C. Bustamante, J. F. Marko, E. D. Siggia, and S. Smith, Science 265, 1599 (1994).
[13] S. Cocco, J. Yan, J.-F. Léger, D. Chatenay, and J. F. Marko, Phys. Rev. E 70, 011910 (2004).

[14] C. Danilowicz, C. Limouse, K. Hatch, A. Conover, V. W. Coljee, N. Kleckner, and M. Prentiss, Proc. Natl. Acad. Sci. U.S.A. 106, 13196 (2009).

[15] G. C. Schatz, Proc. Natl. Acad. Sci. U.S.A. 104, 6885 (2007).

[16] W. K. Olson and P. J. Flory, Biopolymers 11, 25 (1972).

[17] J. O. Tegenfeldt, C. Prinz, H. Cao, S. Chou, W. W. Reisner, R. Riehn, Y. M. Wang, E. C. Cox, J. C. Sturm, P. Silberzan et al., Proc. Natl. Acad. Sci. U.S.A. 101, 10979 (2004).

[18] J. Zlatanova, S. M. Lindsay, and S.H. Leuba, Prog. Biophys. Molec. Biol. 74, 37 (2000).

[19] Y. Zeng, A. Montrichok, and G. Zocchi, Phys. Rev. Lett. 91, 148101 (2003).

[20] G. L. Randall, L. Zechiedrich, and B. M. Pettitt, Nucleic Acids Res. 37, 5568 (2009).

[21] M. Guthold, X. Zhu, C. Rivetti, G. Yang, N. H. Thomson, S. Kasas, H. G. Hansma, B. Smith, P. K. Hansma, and C. Bustamante, Biophys. J. 77, 2284 (1999).

[22] C. Chen, W. Wang, Z. Wang, F. Wei, and X.S. Zhao, Nucleic Acids Res. 35, 2875 (2007).

[23] N. C. Seeman, Nature (London) 421, 427 (2003).

[24] T. Hwa, E. Marinari, K. Sneppen, and L.-H. Tang, Proc. Natl. Acad. Sci. U.S.A. 100, 4411 (2003).

[25] H. Peng and X.S. Ling, Nanotechnology 20, 185101 (2009).

[26] J. Mathé, A. Aksimentiev, D. R. Nelson, K. Schulten, and A. Meller, Proc. Natl. Acad. Sci. U.S.A. 102, 12377 (2005).

[27] J. Rudnick and R. Bruinsma, Phys. Rev. Lett. 94, 038101 (2005).

[28] J. L. Hutter and J. Bechhoefer, Rev. Sci. Instrum. 64, 1868 (1993).

[29] H.-J. Butt and M. Jaschke, Nanotechnology 6, 1 (1995).

[30] G. Mishra, D. Giri, and S. Kumar, Phys. Rev. E 79, 031930 (2009).

[31] J. F. Allemand, D. Bensimon, R. Lavery, and V. Croquette, Proc. Natl. Acad. Sci. U.S.A. 95, 14152 (1998).

[32] M. Rief, H. Clausen-Schaumann, and H.E. Gaub, Nat. Struct. Biol. 6, 346 (1999).

[33] C.P. Calderon, W.-H. Chen, K.-J. Lin, N. C. Harris, and C.-H. Kiang, J. Phys. Condens. Matter 21, 034114 (2009).

[34] S. Whitelam, S. Pronk, and P. L. Geissler, J. Chem. Phys. 129, 205101 (2008).

[35] P. T. X. Li, D. Collin, S. B. Smith, C. Bustamante, and I. Tinoco, Jr., Biophys. J. 90, 250 (2006).

[36] P. Szymczak and M. Cieplak, J. Phys. Condens. Matter 18, L21 (2006).

[37] L. Dougan, G. Feng, H. Lu, and J. M. Fernandez, Proc. Natl. Acad. Sci. U.S.A. 105, 3185 (2008).

[38] B. Schuler, E. A. Lipman, and W. A. Eaton, Nature (London) 419, 743 (2002).

[39] R. B. Best, S. B. Fowler, J. L. Toca-Herrera, and J. Clarke, Proc. Natl. Acad. Sci. U.S.A. 99, 12143 (2002).

[40] D. Watkins, S. Mohan, G. B. Koudelka, and L.D. Williams, J. Mol. Biol. 396, 1145 (2010).

[41] J. Guhaniyogi and G. Brewer, Gene 265, 11 (2001).

[42] Z. Lv, H. Wei, B. Li, and E. Wang, Analyst (Amsterdam) 134, 1647 (2009).

[43] G. Song, C. Chen, X. Qu, D. Miyoshi, J. Ren, and N. Sugimoto, Adv. Mater. 20, 706 (2008).

[44] G. Wang and K. M. Vasquez, Mutat. Res. 598, 103 (2006). 